This item was submitted to Loughborough's Research Repository by the author.

Items in Figshare are protected by copyright, with all rights reserved, unless otherwise indicated.

\title{
Non-uniform deployment of power beacons in wireless powered communication networks
}

PLEASE CITE THE PUBLISHED VERSION

https://doi.org/10.1109/TWC.2019.2899098

PUBLISHER

(C) IEEE

VERSION

AM (Accepted Manuscript)

LICENCE

CC BY-NC-ND 4.0

\section{REPOSITORY RECORD}

Liang, Kai, Liqiang Zhao, Gan Zheng, and Hsiao-Hwa Chen. 2019. "Non-uniform Deployment of Power Beacons in Wireless Powered Communication Networks". figshare. https://hdl.handle.net/2134/37820. 


\title{
NON-UNIFORM DEPLOYMENT OF POWER BEACONS IN WIRELESS POWERED COMMUNICATION NETWORKS
}

Kai Liang, Liqiang Zhao, Gan Zheng, and Hsiao-Hwa Chen ${ }^{\dagger}$, Fellow, IEEE

\author{
${ }^{\dagger}$ Corresponding Author's Address: \\ Hsiao-Hwa Chen \\ Department of Engineering Science \\ National Cheng Kung University \\ 1 Da-Hsueh Road, Tainan City, 70101 Taiwan \\ Tel: +886-6-2757575 ext. 63320 \\ Fax: +886-6-2766549 \\ Email: hshwchendieee.org
}

The paper was submitted on June 24, 2018, revised on November 24, 2018 and accepted on February 4, 2019.

This work was supported in part by China Postdoctoral Science Foundation (2017M613074), Fundamental Research Funds for the Central Universities (JB170102), National Natural Science Foundation of China (61771358), National Natural Science Foundation of Shaanxi Province (2018JM6052), the 111 Project (B08038), the UK EPSRC (EP/N007840/1), and the Leverhulme Trust Research Project Grant (RPG-2017-129).

Kai Liang (email: kliangexidian.edu. cn) and Liqiang Zhao (email: lqzhao@mail.xidian.edu.cn) are with the State Key Laboratory of Integrated Service Networks, Xidian University, China. Gan Zheng (email: g.zheng@lboro.ac.uk) is with the Wolfson School of Mechanical, Electrical, and Manufacturing Engineering, Loughborough University, Loughborough LE11 3TU, U.K. HsiaoHwa Chen (email: hshwchen@ieee.org) is with the Department of Engineering Science, National Cheng Kung University, Taiwan.
Abstract-In wireless powered communication networks (WPCNs), base station (BS) and power beacons (PBs) can offer supplement power for uplink transmission of user equipments (UEs). However, aggregate power consumption of massively deployed PBs may exceed that of a BS. We propose a non-uniform deployment scheme for PBs in WPCNs, where a cell is divided into inner and outer areas, such that BS and PBs can cooperate to power UEs. To be more specific, a BS located in the center of a cell provides downlink power supply for the inner area UEs and uplink information decoding for all the UEs in the cell; while the PBs power UEs in the outer area. With multiple antennas, maximum ratio transmission and maximum ratio combining are adopted for downlink energy beamforming and 
uplink information reception. Considering a finite area of the network, we derive the distribution of the distance from a non-center-located UE to its nearest PB in the outer area. An optimization problem is formulated to minimize total average power consumption, while satisfying BS average transmission power constraint and coverage probability threshold. Moreover, coverage probability is derived for performance evaluation. Numerical results show that the power consumption of the proposed scheme is reduced significantly compared to PB-only WPCNs.

\section{Index Terms-}

Wireless powered communication network; Wireless energy transfer; Non-uniform deployment.

\section{INTRODUCTION}

Radio frequency (RF) wireless energy transfer (WET) has emerged as a key technology to power low energy-consumption Internet of Things (IoT) devices [1]. RF signals can carry both information and energy in wireless powered communication networks (WPCNs), where a base station (BS) transmits energy to and receives information from user equipments (UEs) at the same time. To support information transmission of trillions of low-power wireless devices (such as in wireless sensor networks [2] and wireless body area networks [3]), it is critical to ensure the availability of adequate power at those devices. This prompts a lot of research effort to investigate power transmission schemes in WPCNs.

\section{A. Related Works}

Highly efficient power transmission methods in WPCNs were studied in several existing works. In [4], the authors suggested a joint design of time and power allocation for downlink energy beamforming and uplink information beamforming. In [5], a RFpowered massive multiple input multiple output (MIMO) system adopted slotted transmissions, in which each slot was divided into three phases for channel estimation, downlink power transmission, and uplink data transmission, respectively. In [6], an online power and time allocation algorithm was studied for a WET powered MIMO system with its focus on energy receiving sensitivity with a finite battery capacity. Robust resource allocation methods in non-linear EH model based MIMO WPCNs were investigated in [7]. In [8], we proposed a resource allocation method aiming at profit maximization in WPCNs with a joint consideration of non-linear energy conversion efficiency and battery charge characteristics. In [9], a non-orthogonal multiple access (NOMA) based WPCN was studied with its emphasis on individual data rate maximization and fairness improvement. In [10], the authors focused on energy prediction modeling, which is a key technique to achieve a reliable RF energy harvesting. Different from most of the existing works, the works in [11] investigated energy efficiency optimization issues from a user-centric perspective.

The aforementioned works considered smallsized networks with only two or three transmission pairs. As an effective and tractable analytical method, stochastic geometry, in particular suitable for modeling the distribution of BSs with Poisson point processes (PPPs), has been widely used to analyze the performance of large-scale cellular networks. With the help of the stochastic geometry approach, general models characterizing multicell signal-to-interference-plus-noise ratio (SINR) were proposed in [12], which have been extended to heterogeneous networks [13], device-to-device networks [14], and cooperative networks [15]. The WETs are used for low power consumption devices, such as sensors and wearable devices in IoTs. These ubiquitous devices are normally deployed randomly, and thus we consider spatial randomness of PBs' positions modeled by PPPs.

However, the dependency of downlink and uplink transmissions and different features in WETs and wireless information transmission (WIT) bring in new challenges to analyze large-scaled WPCNs. Accordingly, only very a few works have made their efforts to study the issues on network architecture, power beacon $(\mathrm{PB})$ deployment, and performance analysis of large-scale WPCNs. In [16], the authors studied the architecture, modeling, and deployment of WPCNs that overlay an uplink cellular network with multiple PBs, which charge low power wireless devices by RF WET. Base stations (BSs) and PBs were modeled by homogeneous PPPs. A PB either radiates power isotropically (called isotropic WET) or transmits power directly to its receiver via energy 
beamforming (called directed WET). The tradeoff between the network parameters with an outage constraint was studied. In [17], the authors considered the issues on simultaneous information and energy transfer in large-scaled networks and designed a cooperative protocol with the aid of randomly distributed relays. In [18], the authors analyzed signal to noise ratio (SNR) outage probability and spectral efficiency of a user with a network configuration listed as follows. a) It receives energy from and transmits information to a full-duplex BS; b) It receives energy from symmetrically deployed PBs and transmits energy to a half-duplex BS; and c) It receives energy from and transmits information to symmetrically deployed PBs, which are generated by co-located distributed antenna elements of a BS. In [19], the authors investigated wireless powered sensor networks with randomly deployed PBs and distributed antennas. By modeling the locations of PBs and distributed antennas as PPPs, the outage performance equation of a typical node with a fixed path loss exponent (which equals to four) was derived. In [20], the authors proposed an adaptively directional WET with the aid of antenna arrays in charging regions in a sector, and the maximum received power at a UE was achieved by finding an optimal radius of PBs. In [21], omni-directional and directional energy transmission for wireless powered wearable devices were investigated with a system level performance evaluation.

The distribution of the distance between a UE and its nearest neighbor is still an open issue in stochastic geometry. In [22], the authors derived a distribution of the distance to the $n$-th nearest neighbor in an infinite wireless network, and the result has widely been used in the existing works. In a finite wireless network with a circular shape, the distribution of the distance from the origin to the $n$-th nearest neighbor in binomial point processes and PPPs (conditioned on at least $N$ points in the finite area) was given in [23]. In [24], the authors presented the distribution of an arbitrarily located reference receiver by modeling a finite network as a binomial point process, where the transmitter was chosen by uniform selection or the $k$-closest selection method. The distribution of the distance from one point (not in the origin) to its $n$-th nearest neighbor in a finite wireless relay network was derived in [25], where network extension was adopted to deal with the edge effects in PPPs [26].

\section{B. Motivation and Contributions}

Due to the difference in power reception sensitivities in RF energy harvesting (EH) (about $-10 \mathrm{dBm}$ ) and information decoding (ID) (about $-60 \mathrm{dBm}$ ) [17], the coverage area difference for WET and WIT can be very large. As WET suffers severely from propagation loss, it is difficult for a BS to charge UEs and to receive information from these UEs in a large area. This also creates "doubly near-far" problem in multiple UE systems [27]. The PB, which has a lower transmission power than the BS, can serve as an effective energy supplement to charge UEs by reducing power transmission distance between the PB and UE. However, the power consumption of massively deployed PBs cannot be ignored. For example, if transmission power of a $\mathrm{BS}$ is ten times higher than that of a $\mathrm{PB}$, the power consumption of $10 \mathrm{PBs}$ may be higher than that of a BS due to the basic power consumption (such as circuit power consumption, etc.) of PBs. The same happens to traditional heterogeneous networks, where the power consumption of numerous small cells may exceed that of a BS [28]. Therefore, a joint control in power consumption of BS and PBs is of utmost importance to implement an energy-efficient WET.

Motivated by the aforementioned facts, this paper aims to investigate a non-uniform $\mathrm{PB}$ deployment method in a single cell WPCN, where BS and PBs charge UEs cooperatively in a cell. Specifically, we divide a cell into the inner and the outer areas, where the BS is in charge of the inner cell UEs power supply and all UEs uplink information decoding, and PBs charge the UEs wirelessly in the outer area. The rationale lies on the fact that the BS helps charge the UEs nearby in exchange for significantly reduced power consumption in PBs and the overall network. By adaptively adjusting the sizes of the inner and the outer areas, we can find an energy efficient deployment scheme for PBs. The contributions of this paper can be summarized as follows.

1) We propose a non-uniform deployment scheme for PBs around a BS located in the center of a cell. The UEs can receive power from either the BS or PBs with multiple antennas in a directional manner, depending on their locations (in the inner or the outer area). We take the advantages of multiple antennas to facilitate downlink energy beamforming through maximum ratio transmission (MRT) 
precoder and uplink information reception by maximum ration combining (MRC) receiver.

2) Considering a finite area of a wireless powered network, we derived the distribution of the distance between a non-center-located UE and its nearest PB in the outer area with an annular shape, conditioned on at least $N_{1} \mathrm{PBs}$ existed in this area.

3) We design a quality of service (QoS) aware deployment method and derive the total average power consumption in a WPCN. Here, the QoS refers to a satisfactory coverage probability, which is defined as the probability that a UE's uplink rate is above a given threshold. The problem is formulated as a function of the inner area's radius to minimize total average power consumption in a cell, while satisfying the BS transmit power constraint and UEs' coverage probability constraint.

4) We evaluate the system performance (i.e., coverage probability) under the assumption of receiving energy from the nearest power providers by discretizing the transmission distances of the uplink in the outer area.

The rest of the paper can be outlined as follows. The system model is provided in Section II. In Section III, the distributions of transmission distances, a QoS-aware deployment method, and coverage probability are derived. In Section IV, numerical results are given to show the performance of the proposed deployment method, followed by the conclusions of this paper in Section V.

\section{SYSTEM MODEL}

Let us consider a circular cell centered at point $O$ with its radius $R$, which includes a BS, several PBs, and $K$ UEs, as shown in Fig. 1. The BS and each of PBs are equipped with $N_{t}$ antennas and each of UEs is equipped with a single antenna, respectively. Let $B(o, x)$ denote a circle centered at point $o$ with its radius $x$. The cell can be denoted as $A=B(o, R)$. The BS is located at the cell center $o$ and is in charge of downlink WET for the UEs in the inner area and performs uplink information reception from all UEs, where the inner area is defined as a circle with its radius $D$ from the $\mathrm{BS}$, i.e., $A_{\text {in }}=B(0, D)$. PBs are deployed following a two-dimensional homogeneous PPP with its density $\lambda_{p}$, which can be

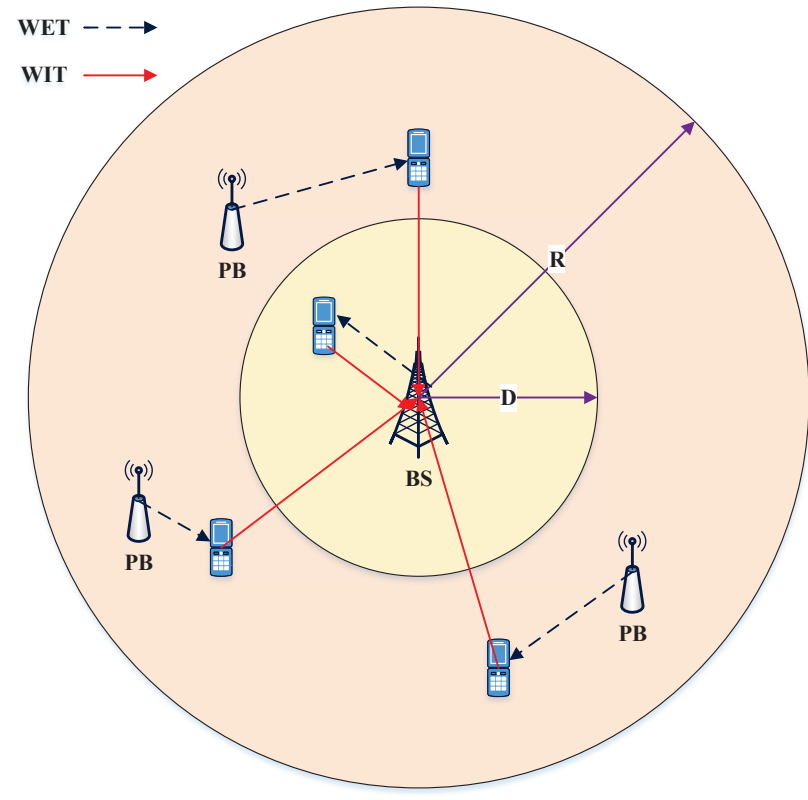

Figure 1. A system model considered in this paper.

denoted as $\Phi(A)$, and those which are located in the inner region are inactive. $K$ UEs each equipped with a single antenna are independently and uniformly distributed in the whole area. Denote the outer area as $A_{\text {out }}$. The probabilities of a UE $u$ located in the inner and the outer regions are given as

$$
\begin{aligned}
& \operatorname{Pr}\left(u \in A_{\text {in }}\right)=\frac{\left|A_{\text {in }}\right|}{|A|}=\left(\frac{D}{R}\right)^{2}, \\
& \operatorname{Pr}\left(u \in A_{\text {out }}\right)=\frac{\left|A_{\text {out }}\right|}{|A|}=\frac{R^{2}-D^{2}}{R^{2}} .
\end{aligned}
$$

Each power provider (BS or PBs) wirelessly charging multiple UEs can form a power cell. Denote a set of power cells as $\Psi=\left\{\Psi_{i}\right\}$, where $i$ denotes the $i$-th power cell. Specifically, $i=0$ and $i \geq 1$ represent the UEs being associated with the $\mathrm{BS}$ and PBs in the power cell.

The main task of this work is to minimize the total average power consumption $\mathbf{E}\left(P_{t o t}\right)$ of the network, while satisfying per-UE QoS requirement (i.e., coverage probability) by adaptively adjusting the inner area radius $D$. In the text followed, we first formulate an optimization problem. Let us define the coverage probability by the probability that the uplink rate of a UE is higher than a certain threshold, i.e., $t$ bits/s. The corresponding problem 
is formulated as

$$
\begin{array}{rl}
\min _{D} & \mathbf{E}\left(P_{t o t}\right), \\
\text { s.t. } & C 1: \operatorname{Pr}\left(r_{i u}>t\right) \geq \varepsilon, \quad \forall u, i, \\
& C 2: \mathbf{E}\left(P_{0}\right) \leq P_{b m},
\end{array}
$$

where $r_{i u}$ is the uplink rate of the $u$-th UEs in the $i$-th power cell, $C 1$ denotes that the coverage probability should be kept above the threshold $\varepsilon \in[0,1]$, and $C 2$ denotes the maximal transmission power $P_{b m}$ constraint of the BS. In this optimization problem, it is assumed that in a benchmark PB-only charging method (in which the BS is not involved in charging UEs, i.e., $D=0$ ), UEs' QoS requirement can be satisfied. This means that PB transmission power will not exceed its transmission power limit; otherwise the PBs in the outer area cannot ensure their QoS requirements in the case of $D>0$, due to the uniform distribution of UEs' locations. The PBs' power constraint actually is an inactive constraint, and thus we omit it in our problem formulation.

We adopt a "harvest-then-transmit" protocol [27] in the following analysis. Two adjacent slots are used in transmission process, where the first slot is used for downlink WET and the second slot is for uplink WIT. The BS and PBs are equipped with multiple antennas to facilitate directional power transfer via energy beamforming. The transmit power of a power provider is distributed evenly across a number of UEs, which are powered by this power provider. The received power at a UE $u \in \Psi_{i}$ is given as

$$
\begin{aligned}
P_{i u}= & \frac{P_{i}}{K_{i}} \Theta_{i u}^{-\alpha}\left(\left|\mathbf{h}_{i, i_{u}}^{H} \mathbf{v}_{i, i_{u}}\right|^{2}+\sum_{n \neq u}\left|\mathbf{h}_{i, i_{u}}^{H} \mathbf{v}_{i, i_{n}}\right|^{2}\right) \\
& +\sum_{b \neq i} \frac{P_{b}}{K_{b}} \Theta_{b u}^{-\alpha} \sum_{u=1}^{K_{b}}\left|\mathbf{h}_{b, i_{u}}^{H} \mathbf{v}_{b, b_{u}}\right|^{2},
\end{aligned}
$$

where $K_{i}$ denotes the number of UEs in the $i$ th power cell, and $P_{i}$ stands for the transmission power of the $i$-th power provider. $\mathbf{h}_{b, i_{u}}$ represents a downlink channel gain between power provider $b$ and the $u$-th UE in the $i$-th power cell, and the downlink channel suffers independently Rayleigh fading with zero mean and unit variance. $\mathbf{v}_{b, b_{u}}$ is the $b$-th power provider's precoder for its $u$-th UE. We adopt maximum ratio transmission (MRT) precoding at BS and PBs, namely $\mathbf{v}_{b, b_{u}}=\frac{\mathbf{h}_{b, b_{u}}}{\left\|\mathbf{h}_{b, b_{u}}\right\|_{2}}$, which is a frequently used precoding scheme for energy beamforming [29, 30]. Denote $y_{i u}=\left|\mathbf{h}_{i, i_{u}}^{H} \mathbf{v}_{i, i_{u}}\right|^{2}+$ $\sum_{n \neq u}\left|\mathbf{h}_{i, i_{u}}^{H} \mathbf{v}_{i, i_{n}}\right|^{2}$ as joint channel power fading from the nearest power provider to the UE. $\Theta_{i u}^{-\alpha}$ is the path loss from a UE to its power provider. Let the path loss exponent for WET be $\alpha>0$.

Because WET has a very short effective transmission distance (e.g., 10 meters) and energy beamforming facilitates energy harvesting received from the nearest dedicated power provider, the amount of power received from non-nearest power providers is very tiny. Besides, it is very difficult to take into account both energy beam forming and receiving power from multiple power providers. To reduce analysis complexity, following the ways suggested in the literatures $[16,18]$, we only consider that a UE receives the power from its nearest power provider in this paper. The issues that a UE may receive power from multiple power providers will be investigated in our future works. Under this assumption, the received power at UE $u \in \Psi_{i}$ can be calculated by

$$
P_{i u}=\frac{P_{i}}{K_{i}} \Theta_{i u}^{-\alpha} y_{i u}
$$

The power of received signal at the $\mathrm{BS}$ is given by

$$
P_{b u}=\xi \zeta P_{i u} \Theta_{0 u}^{-\beta} g_{0 u},
$$

where $\xi$ denotes the RF-DC conversion efficiency [31]. A UE cannot allocate all harvested energy for uplink transmission because of circuit power consumption and energy storage losses in the UE. We assume that a fraction of harvested energy $\zeta$ is consumed for uplink transmission, and the same assumption was made in $[18,32] . g_{0 u}$ and $d_{0 u}$ denote the channel power fading and the distance between the UE and the BS, respectively. The path loss exponent for WIT is $\beta$. Denote $d_{i u}$ as the transmission distance from a UE to the $i$-th power provider. Specifically, $d_{0 u}$ and $d_{1 u}$ are the distance between a UE and the BS, and the distance between a UE in the outer area $\left(u \in A_{\text {out }}\right)$ and its nearest $\mathrm{PB}$, respectively. Denote $s \in\{\alpha, \beta\}$, and then path loss $\Theta_{i u}^{-s}$ is defined as [28]

$$
\Theta_{i u}^{-s}= \begin{cases}\theta^{-1}\left(\frac{d_{i u}}{d_{0}}\right)^{-s}, & d_{i u}>d_{0} \\ \theta^{-1}, & d_{i, u} \leq d_{0}\end{cases}
$$

where $d_{0}$ denotes a reference distance. For discussion simplicity, let us set the reference distance as $d_{0}=1 \mathrm{~m} . \theta^{-1} \in\left\{\theta_{E}^{-1}, \theta_{I}^{-1}\right\}$ denotes a fixed path 
loss, where $\theta_{E}^{-1}$ and $\theta_{I}^{-1}$ represent the fixed path loss exponents for WET and WIT, respectively.

Assume that all UEs equally share the whole uplink bandwidth $W$ [28]. This means that frequency division multiple access (FDMA) is adopted in uplink information transmission. As FDMA is adopted for multi-user interference avoidance, we adopt MRC at the BS in uplink information reception. Therefore, the uplink data rate of UE $u \in \Phi_{i}$ is given by

$$
r_{i u}=\frac{W}{K} \log _{2}\left(1+\frac{P_{b u}}{W \sigma^{2} / K}\right) .
$$

Denote $Z=y_{i u} g_{0 u}$. According to [33], we have $y_{i u} \sim \Gamma\left(N_{t}+K_{i}-1,1\right)$ and $g_{0 u} \sim \Gamma\left(N_{t}, 1\right)$, where $\Gamma(k, b)$ denotes a Gamma distribution with a shape parameter $k$ and a scale parameter $b$. Since WET and WIT account for two slots, $y_{i u}$ and $g_{0 u}$ are independent, and thus the probability density function (PDF) of $Z$ can be written as

$$
\begin{aligned}
f_{Z}(z) & =\int_{-\infty}^{+\infty} \frac{1}{|x|} f_{y_{i u}}(x) f_{g_{0 u}}\left(\frac{z}{x}\right) d x \\
& =\frac{2 z^{\frac{1}{2}\left(2 N_{t}+K i-3\right)} \mathcal{K}_{1-K_{i}}(2 \sqrt{z})}{\Gamma\left(N_{t}\right) \Gamma\left(N_{t}+K_{i}-1\right)},
\end{aligned}
$$

where $\mathcal{K}_{n}(z)$ is the modified Bessel function of the second kind, $n$ is the order of this function, and $\Gamma(\cdot)$ denotes a Gamma function.

Denote ${ }_{p} F_{q}\left(a_{1}, \cdots, a_{p} ; b_{1}, \cdots, b_{q} ; z\right)$ as the hypergeometric function, and the regularized hypergeometric function is defined as ${ }_{p} \tilde{F}_{q}\left(a_{1}, \cdots, a_{p} ; b_{1}, \cdots, b_{q} ; z\right)=\frac{p F_{q}\left(a_{1}, \cdots, a_{p} ; b_{1}, \cdots, b_{q} ; z\right)}{\Gamma\left(b_{1}\right) \cdots \Gamma\left(b_{q}\right)}$. Then, the cumulative distribution function (CDF) of $Z$ is written as

$$
\begin{aligned}
F_{Z}(z)= & \int_{-\infty}^{z} f_{Z}(u) d u=\pi z^{N_{t}} \csc \left(K_{i} \pi\right) \times \\
& {\left[\frac{z^{K_{i}-1}{ }_{1} \tilde{F}_{2}\left(N_{t}+K_{i}-1 ; K_{i}, N_{t}+K_{i} ; z\right)}{\Gamma\left(N_{t}\right)}\right.} \\
& \left.-\frac{{ }_{1} \tilde{F}_{2}\left(N_{t} ; 2-K_{i}, 1+N_{t} ; z\right)}{\Gamma\left(N_{t}+K_{i}-1\right)}\right] .
\end{aligned}
$$

The power consumption of a BS includes two parts, which starts from a basic level $\underline{P}$ and increases with its downlink transmission power $P_{0}$, or

$$
P_{b}=\underline{P}+P_{0}(D),
$$

where $P_{0}(D)$ indicates that $P_{0}$ is a function of the inner cell radius $\mathrm{D}$. Likewise, the power consumption of a PB includes a basic level $p$ and its downlink transmission power $P_{i}$ as

$$
P_{p}=\underline{p}+P_{i}(D), \quad i=1, \cdots, N .
$$

Therefore, the total average power consumption of a cell is calculated by

$$
\mathbf{E}\left(P_{t o t}\right)=\underline{P}+\mathbf{E}(N) \underline{p}+\mathbf{E}\left[P_{T}(D)\right],
$$

where $\mathbf{E}\left[P_{T}(D)\right]$ is the total average downlink transmission power, which is derived in the section followed. $N$ refers to the number of PBs.

\section{QOS-AWARE NON-UNIFORM PB}

\section{DEPLOYMENT}

\section{A. PDFs of Transmission Distances}

As UEs are uniformly distributed, the PDF of $d_{0 u}$ is given by

$$
f_{d_{0 u}}(r)=\frac{2 r}{R^{2}}, \quad 0 \leq r \leq R .
$$

According to the law of total probability, the PDFs of $d_{0 u}$ conditioned on the inner and the outer areas can be written as

$$
\begin{aligned}
f_{d_{0 u} \mid u \in A_{\text {in }}}(r) & =\frac{2 r}{D^{2}}, \quad 0 \leq r \leq D, \\
f_{d_{0 u} \mid u \in A_{\text {out }}}(r) & =\frac{2 r}{R^{2}-D^{2}}, \quad D \leq r \leq R .
\end{aligned}
$$

The PDF of the distance $d_{1 u}$ between a UE and its nearest $\mathrm{PB}$ is very difficult to be derived due to the following reasons. First, the PBs are located in a finite area (namely, the outer area with an annular shape) such that the analytical results in an infinite network scenario cannot be used. Second, the UE is not located at the origin, making $\operatorname{PPP} \Phi_{p}\left(A_{\text {out }}\right)$ anisotropic from the viewpoint of a UE. Third, the shape of annular outer area forces us to consider the edge effects on both the whole cell edge and the inner area edge. Inspired by the works done in [23], next we derive a complementary cumulative distribution function (CCDF) of $d_{1 u}$.

Denote $b\left(d_{0 u}, r\right)$ as an intersecting region between a circle $B\left(d_{0 u}, r\right)$ and the outer area, which is centered at $d_{0 u}$ with its radius $r$. Note that $b\left(d_{0 u}, r\right)$ 


$$
\begin{gathered}
S(a, b)=a^{2} \arccos \left(\frac{d_{0 u}^{2}+a^{2}-b^{2}}{2 d_{0 u} a}\right)+b^{2} \arccos \left(\frac{d_{0 u}^{2}+b^{2}-a^{2}}{2 d_{0 u} b}\right) \\
-0.5 \sqrt{\left(-d_{0 u}+a+b\right)\left(d_{0 u}+a-b\right)\left(d_{0 u}-a+b\right)\left(d_{0 u}+a+b\right)} . \\
\nu= \begin{cases}\pi r^{2}, & \text { if } 0 \leq r \leq \min \left(d_{0 u}-D, R-d_{0 u}\right), \\
\pi r^{2}-S(D, r), & \text { if } d_{0 u}-D<r \leq R-d_{0 u}, r<d_{0 u}+D, \\
S(R, r), & \text { if } R-d_{0 u}<r \leq d_{0 u}-D, \\
S(R, r)-S(D-r), & \text { if } \max \left(d_{0 u}-D, R-d_{0 u}\right)<r<d_{0 u}+D, \\
\pi\left(r^{2}-D^{2}\right), & \text { if } d_{0 u}+D \leq r \leq R-d_{0 u}, \\
S(R, r)-\pi D^{2}, & \text { if } \max \left(d_{0 u}+D, R-d_{0 u}\right) \leq r<d_{0 u}+R, \\
\pi\left(R^{2}-D^{2}\right), & \text { if } r \geq d_{0 u}+R .\end{cases}
\end{gathered}
$$

may not have a circular shape. Since the cell is rotation invariant around the origin, the rotation angle is not considered. Denote the intersecting area of two circles $B(0, a)$ and $B\left(d_{0 u}, b\right)$ as $S(a, b)$, which can be calculated by Eqn. (16).

When the outer area has at least $N_{1} \mathrm{PBs}$, the CCDF of the distance from a UE to its nearest PB can be obtained by following theorem.

Theorem 1. When there are at least $N_{1} P B s$ in the outer area, the CCDF of the distance from a UE to its nearest $P B d_{1 u}$ is expressed by

$$
\bar{F}_{d_{1 u} \mid N_{1}}(r)=\frac{e^{-\lambda_{p} \nu}\left[1-\sum_{k=0}^{N_{1}-1} e^{-\lambda_{p} \nu^{\prime}}\left(\lambda_{p} \nu^{\prime}\right)^{k} / k !\right]}{\sum_{k=N_{1}}^{\infty} e^{-\lambda_{p} \pi\left(R^{2}-D^{2}\right)}\left[\lambda_{p} \pi\left(R^{2}-D^{2}\right)\right]^{k} / k !} .
$$

$\nu^{\prime}$ and $\nu$ can be obtained as

$$
\nu^{\prime}=\pi\left(R^{2}-D^{2}\right)-\nu
$$

\section{Proof: See Appendix A.}

Thus, the PDF of $d_{1 u}$ is given by

$$
f_{d_{1 u} \mid N_{1}}(r)=-\frac{\partial \bar{F}_{d_{1 u} \mid N_{1}}(r)}{\partial r}, \quad u \in A_{\text {out }} \text {. }
$$

Based on (20), Fig. 2 depicts the CDFs and PDFs of the distance from a UE to its nearest $\mathrm{PB}$ in the outer area, where $D=30 \mathrm{~m}, d_{0 u}=40 \mathrm{~m}$, and $N_{1}$ ranges from 2 to 20 . In a PB-only powered scenario $(D=0)$, the CCDF of the distance between a UE and its nearest PB can be calculated as follows.

Corollary 1. In a PB-only powered scenario, the CCDF of $d_{1 u}$ conditioned on at least $N_{1}$ PBs in the cell is given by

$$
\bar{F}_{d_{1 u} \mid N_{1}}(r)=\frac{e^{-\lambda_{p} \nu}\left[1-\sum_{k=0}^{N_{1}-1} \frac{e^{-\lambda_{p} \nu^{\prime}}\left(\lambda_{p} \nu^{\prime}\right)^{k}}{k !}\right]}{\sum_{k=N_{1}}^{\infty} \frac{e^{-\lambda_{p} \pi R^{2}}\left(\lambda_{p} \pi R^{2}\right)^{k}}{k !}},
$$

where $\nu$ ' and $\nu$ can be obtained as

$$
\nu^{\prime}=\pi R^{2}-\nu
$$




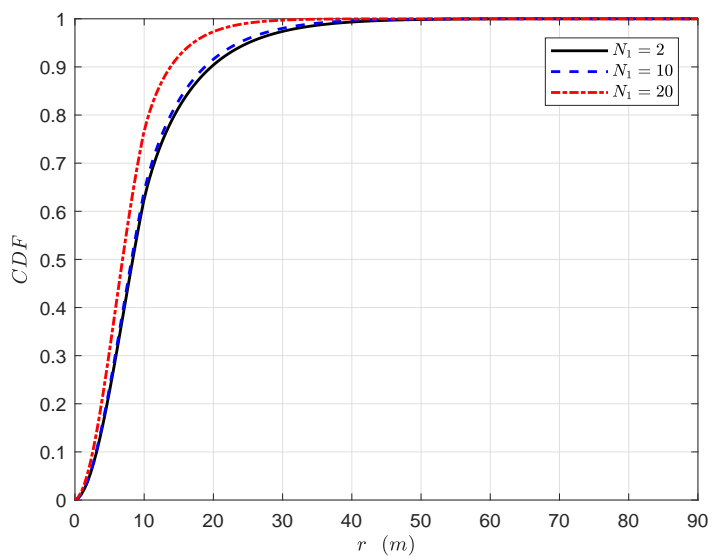

(a) $\mathrm{CDF}$

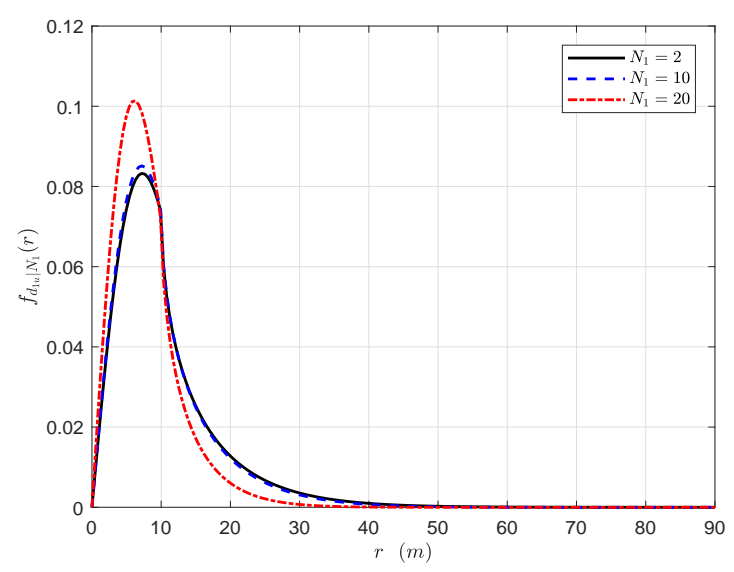

(b) PDF

Figure 2. Distributions of $d_{1 u}$, where $d_{0 u}=40$ and $D=30$.

$$
\nu= \begin{cases}\pi r^{2}, & \text { if } 0 \leq r \leq R-d_{0 u}, \\ S(R, r), & \text { if } R-d_{0 u}<r \leq R+d_{0 u}, \\ \pi R^{2}, & \text { if } r \geq d_{0 u}+R .\end{cases}
$$

\section{B. QoS-Aware Power Transmission}

Since all UEs share the whole uplink bandwidth equally, the coverage probability for $\mathrm{UE} u \in \Psi_{i}$ is given by

$$
\begin{aligned}
C & =\operatorname{Pr}\left(r_{i u}>t\right) \\
& =\operatorname{Pr}\left[\frac{W}{K} \log _{2}\left(1+\frac{P_{b u}}{W \sigma^{2} / K}\right)>t\right] \\
& =\operatorname{Pr}\left[y_{u i} g_{0 u}>\left(2^{\frac{K t}{W}}-1\right) \frac{W \sigma^{2}}{\xi \zeta K P_{i}} K_{i} \Theta_{i u}^{\alpha} \Theta_{0 u}^{\beta}\right] \\
& =1-\pi Z_{0}^{N_{t}} \csc \left(K_{i} \pi\right) \\
& \times\left[\frac{Z_{0}^{K_{i}-1}{ }_{1} \tilde{F}_{2}\left(N_{t}+K_{i}-1 ; K_{i}, N_{t}+K_{i} ; Z_{0}\right)}{\Gamma\left(N_{t}\right)}\right. \\
& \left.-\frac{{ }_{1} \tilde{F}_{2}\left(N_{t} ; 2-K_{i}, 1+N_{t} ; Z_{0}\right)}{\Gamma\left(N_{t}+K_{i}-1\right)}\right],
\end{aligned}
$$

where we have

$$
Z_{0}=\left(2^{\frac{K t}{W}}-1\right) \frac{W \sigma^{2}}{\xi \zeta K P_{i}} K_{i} \Theta_{i u}^{\alpha} \Theta_{0 u}^{\beta}
$$

The QoS requirement of a UE is satisfied if its coverage probability is larger than a given threshold $\varepsilon$, i.e., or

$$
C \geq \varepsilon
$$

However, it is difficult to get an explicit equation with respect to $P_{i}$. Fortunately, $C$ is a monotonously decreasing function with $Z_{0}$, due to the property of CDF. Thus, (26) can be solved by a bisection method. There is only one problem to solve numerically (26), i.e., the number of UEs $K_{i}$ served by $\Psi_{i}$ is a random variable. For simplicity, we use the mean value $\mathbf{E}\left(K_{i}\right)$ to replace $K_{i}$, which is calculated in the next subsection, and thus $C$ can be approximated by

$$
\tilde{C}=\left.C\left(Z_{0}, K_{i}\right)\right|_{K_{i}=\mathbf{E}\left(K_{i}\right)} .
$$

Denoting the solution of $\tilde{C}=\varepsilon$ as $Z_{0}=Z_{0}^{*}$, we have

$$
P_{i}=A_{i} \frac{K_{i}}{K} \Theta_{i u}^{\alpha} \Theta_{0 u}^{\beta}, \quad i=0, \cdots, N,
$$

where

$$
A_{i}=\frac{\left(2^{\frac{K t}{W}}-1\right) W \sigma^{2}}{\xi \zeta Z_{0}^{*}}, \quad i=0, \cdots, N .
$$




\section{Average Transmitted Power}

Denote average downlink transmission powers for the inner and the outer areas as $P_{i n}$ and $P_{\text {out }}$, respectively. Assume that UEs are located in the inner and the outer areas with their probabilities given in (1) and (2), respectively. Next, we derive the average total downlink transmission power of the network $\mathbf{E}\left(P_{T}(D)\right)$, which is expressed as

$$
\begin{aligned}
\mathbf{E}\left[P_{T}(D)\right]= & P_{\text {in }}+P_{\text {out }} \\
= & \mathbf{E}_{u \in A_{\text {in }}}\left(P_{0}\right)\left(\frac{D}{R}\right)^{2} \\
& +\mathbf{E}_{u \in A_{\text {out }}}\left(N P_{i}\right) \frac{R^{2}-D^{2}}{R^{2}} .
\end{aligned}
$$

There are three random variables (RVs) in $P_{0}$ and $P_{i}$, namely $K_{i}, \Theta_{i u}^{\alpha}$, and $\Theta_{0 u}^{\beta}$, where $K_{i}$ is independent of $\Theta_{i u}^{\alpha}$ and $\Theta_{0 u}^{\beta}$. Next, we will calculate the expectation of $K_{i}$.

Assume that $K$ UEs exist in the whole area $A$. The number of UEs in a subarea $\tilde{A} \in A$ follows a binomial distribution $\mathcal{B}\left(K, \frac{|\tilde{A}|}{|A|}\right)$. Therefore, the probability that there are $n$ UEs in the inner area can be calculated as

$$
\begin{aligned}
& \operatorname{Pr}\left(K_{0}=n \mid \text { Number of UEs }=K\right) \\
= & \left(\begin{array}{c}
K \\
n
\end{array}\right)\left(\frac{D}{R}\right)^{2 n}\left[1-\left(\frac{D}{R}\right)^{2}\right]^{K-n},
\end{aligned}
$$

and the corresponding expectation is given by

$$
\mathbf{E}_{K_{0} \mid K, u \in A_{i n}}\left(K_{0}\right)=K\left(\frac{D}{R}\right)^{2}
$$

where $\left(\begin{array}{l}K \\ n\end{array}\right)=\frac{K !}{n !(K-n) !}$ is the combinatorial number and refers to the operation selecting $n$ items out of $K$ items, and $K$ ! represents the factorial of $K$.

When calculating the expectation of $K_{0}$, we can derive $\mathbf{E}_{u \in A_{\text {in }}}\left(P_{0}\right)$, which is given as

$$
\begin{aligned}
& \mathbf{E}_{u \in A_{i n}}\left(P_{0}\right) \\
= & \mathbf{E}_{u \in A_{i n}}\left(\frac{A_{0}}{K} K_{0} \Theta_{0 u}^{\alpha+\beta}\right) \\
= & \frac{A_{0}}{K} \mathbf{E}_{K_{0} \mid K, u \in A_{i n}}\left(K_{0}\right) \mathbf{E}\left(\Theta_{0 u}^{\alpha+\beta}\right) \\
= & \frac{A_{0}}{K} \mathbf{E}_{K_{0} \mid K, u \in A_{i n}}\left(K_{0}\right) \int_{0}^{D} \Theta_{0 u}^{\alpha+\beta} f_{d_{0 u \mid u \in A_{i n}}}(r) d r \\
= & \frac{A_{0}}{K} \mathbf{E}_{K_{0} \mid K, u \in A_{i n}}\left(K_{0}\right) \\
& \times\left(\int_{0}^{1} \theta_{E} \theta_{I} \frac{2 r}{D^{2}} d r+\int_{1}^{D} \theta_{E} \theta_{I} \frac{2 r^{\alpha+\beta+1}}{D^{2}} d r\right) \\
= & \frac{A_{0} \theta_{E} \theta_{I}\left(2 D^{\alpha+\beta+2}+\alpha+\beta\right)}{R^{2}(\alpha+\beta+2)} .
\end{aligned}
$$

Therefore, the average downlink transmission power of the inner area is

$$
\begin{aligned}
P_{\text {in }} & =\mathbf{E}_{u \in A_{i n}}\left(P_{0}\right)\left(\frac{D}{R}\right)^{2} \\
& =\frac{A_{0} \theta_{E} \theta_{I} D^{2}\left(2 D^{\alpha+\beta+2}+\alpha+\beta\right)}{R^{4}(\alpha+\beta+2)} .
\end{aligned}
$$

To calculate the probability of the number of UEs powered by a $\mathrm{PB}$, we have to know the area of a power cell first. Since the density of PBs is $\lambda_{p}$, the average area of a power cell is $\frac{1}{\lambda_{p}}$. Denote $V$ as the charging area of a $\mathrm{PB}$ and $\hat{V}=\lambda_{p} V$ as the normalized cell area. According to [34], the PDF of $\hat{V}$ is written as

$$
f_{\hat{V}}(\hat{v})=\frac{(3.5)^{3.5}}{\Gamma(3.5)} \hat{v}^{2.5} e^{-3.5 \hat{v}}
$$

The conditional probability that there are $n$ UEs in a power cell is given by

$$
\begin{aligned}
& \operatorname{Pr}\left(K_{i}=n \mid \text { Number of UEs }=K, V=v\right) \\
= & \left(\begin{array}{c}
K \\
n
\end{array}\right)\left(\frac{v}{\pi R^{2}}\right)^{n}\left(1-\frac{v}{\pi R^{2}}\right)^{K-n},
\end{aligned}
$$

and its corresponding expectation is

$$
\mathbf{E}_{K_{i} \mid K, V=v, u \in A_{\text {out }}}\left(K_{i}\right)=\frac{K v}{\pi R^{2}} .
$$


Given the total number of UE $K$, the average number of UEs powered by a PB can be obtained by

$$
\begin{aligned}
& \mathbf{E}_{K_{i} \mid K, u \in A_{\text {out }}}\left(K_{i}\right)=\int_{0}^{\pi \lambda_{p}\left(R^{2}-D^{2}\right)} K \frac{v}{\pi R^{2}} f_{\hat{V}}(\hat{v}) d \hat{v} \\
= & \frac{K}{\pi R^{2} \lambda_{p}}\left\{1-0.0859717 \Gamma\left[4.5,3.5 \pi \lambda_{p}\left(R^{2}-D^{2}\right)\right]\right\}
\end{aligned}
$$

where the upper incomplete Gamma function is defined as $\Gamma(s, x)=\int_{x}^{\infty} t^{s-1} e^{-t} d t$.

Next, we can derive the transmission power in the outer area as

$$
\begin{aligned}
& \mathbf{E}_{u \in A_{\text {out }}}\left(N P_{i}\right)=\mathbf{E}_{u \in A_{\text {out }}}\left(\frac{A_{1}}{K} N K_{i} \Theta_{i u}^{\alpha} \Theta_{0 u}^{\beta}\right) \\
= & \frac{A_{1}}{K} \mathbf{E}(N) \mathbf{E}_{K_{i} \mid K, u \in A_{\text {out }}}\left(K_{i}\right) \mathbf{E}\left(\Theta_{i u}^{\alpha} \Theta_{0 u}^{\beta}\right) .
\end{aligned}
$$

The number of PBs in the outer area is given by

$$
\mathbf{E}(N)=\pi\left(R^{2}-D^{2}\right) \lambda_{p} .
$$

$\mathbf{E}\left(\Theta_{i u}^{\alpha} \Theta_{0 u}^{\beta}\right)$ can be written as

$$
\begin{aligned}
& \quad \mathbf{E}\left(\Theta_{i u}^{\alpha} \Theta_{0 u}^{\beta}\right) \\
& =\int_{D}^{R} \theta_{I} z^{\beta} f_{d_{0 u} \mid u \in A_{\text {out }}}(z)\left[\int_{0}^{1} \theta_{E} f_{d_{1 u} \mid N_{1}}(r) d r\right. \\
& \left.\quad+\int_{1}^{z+R} \theta_{E} r^{\alpha} f_{d_{1 u} \mid N_{1}}(r) d r\right] d z .
\end{aligned}
$$

Since the power of a BS is much larger than that of a $\mathrm{PB}$, we assume that the radius of the inner area is $D>1$ in above derivation. The closed form expression of $\mathbf{E}\left(\Theta_{i u}^{\alpha} \Theta_{0 u}^{\beta}\right)$ is extremely complicated, and we will obtain $\mathbf{E}\left(\Theta_{i u}^{\alpha} \Theta_{0 u}^{\beta}\right)$ by numerical methods.

Thus, the power consumptions of a PB and that in the outer area are given by Eqn. (42) and

$$
\begin{aligned}
P_{\text {out }} & =\mathbf{E}\left(P_{i}\right) \mathbf{E}(N) \operatorname{Pr}\left(u \in A_{\text {out }}\right) \\
& =\frac{\pi\left(R^{2}-D^{2}\right)^{2} \lambda_{p}}{R^{2}} \mathbf{E}\left(P_{i}\right) .
\end{aligned}
$$

\section{Adaptive Deployment Scheme}

Assume that, when $D$ equals zero, the density of PBs is sufficient to charge all UEs to satisfy the coverage probability threshold. Then, with a given QoS demand, i.e., $\operatorname{Pr}\left(\frac{W}{K} \log _{2}\left(1+\frac{P_{b r}}{W \sigma^{2} / K}\right)>\right.$ $t) \geq \varepsilon$, we can formulate the problem as the way to minimize the power consumption of a network, while satisfying the power constraints of the BS. Therefore, the problem (3) can be recast as

$$
\begin{gathered}
\min _{D} \mathbf{E}\left[P_{T}(D)\right]+\underline{P}+\underline{p} \lambda_{p} \pi\left(R^{2}-D^{2}\right), \\
\text { s.t. } \mathbf{E}\left[P_{0}(D)\right] \leq P_{b m}, \quad 0 \leq D \leq R .
\end{gathered}
$$

The convexity of the objective function of the problem (44) is difficult to be demonstrated analytically. Fig. 3 shows the relationship between the power consumption and inner area radius $D$, and we can find that the total power consumption is a unimodal function with respect to $D$, where $D$ ranges from 1 to the maximal radius $d_{\max }$ of the inner area. Given the maximal transmission power of the BS $P_{b m}$, we can find the maximal radius $d_{\max }$ of the inner area by solving the inequality of the constraint. Thus, problem (44) can be solved iteratively by one-dimensional search, i.e., Golden section method, as shown in Algorithm 1.

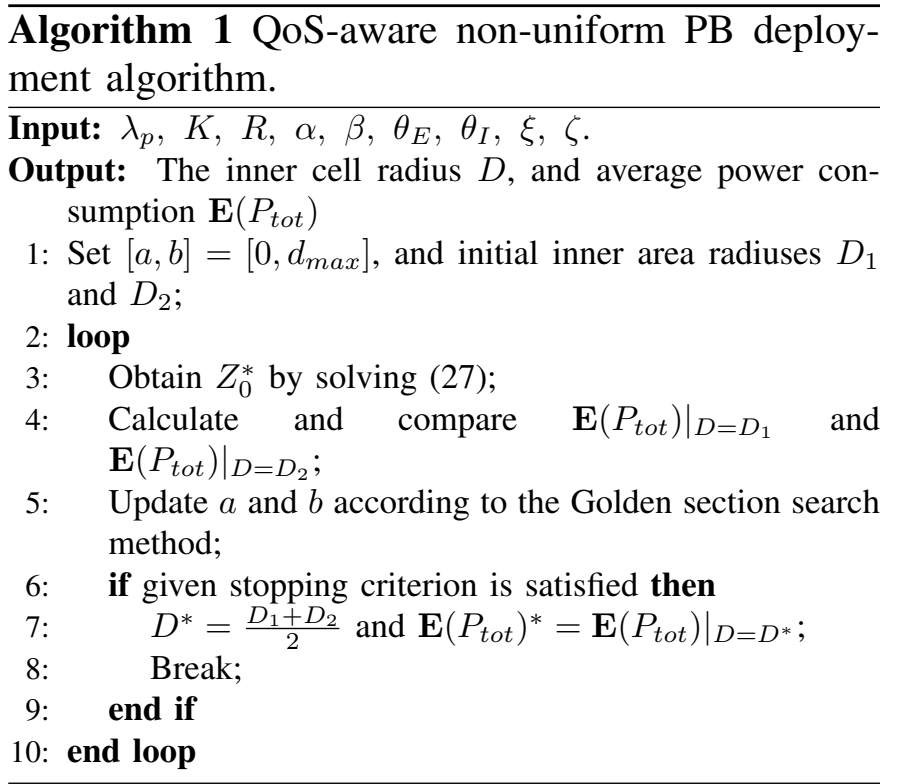

\section{E. Coverage Probability}

In this subsection, we will derive the coverage probability of a typical UE $u$. Different from the previous analysis, here we assume that the network 


$$
\begin{aligned}
\mathbf{E}\left(P_{i}\right)= & \frac{A_{1}}{\pi R^{2} \lambda_{p}}\left\{1-0.0859717 \Gamma\left[4.5,3.5 \pi \lambda_{p}\left(R^{2}-D^{2}\right)\right]\right\} \\
& \times \int_{D}^{R} \theta_{I} z^{\beta} f_{d_{0 u} \mid u \in A_{\text {out }}}(z)\left[\int_{0}^{1} \theta_{E} f_{d_{1 u} \mid N_{1}}(r) d r+\int_{1}^{z+R} \theta_{E} r^{\alpha} f_{d_{1 u} \mid N_{1}}(r) d r\right] d z,
\end{aligned}
$$

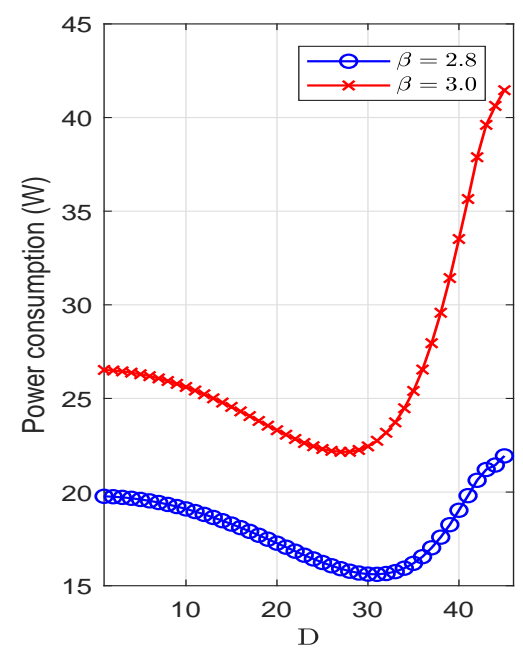

(a) $\lambda_{p}=0.003 / m^{2}, K=60$

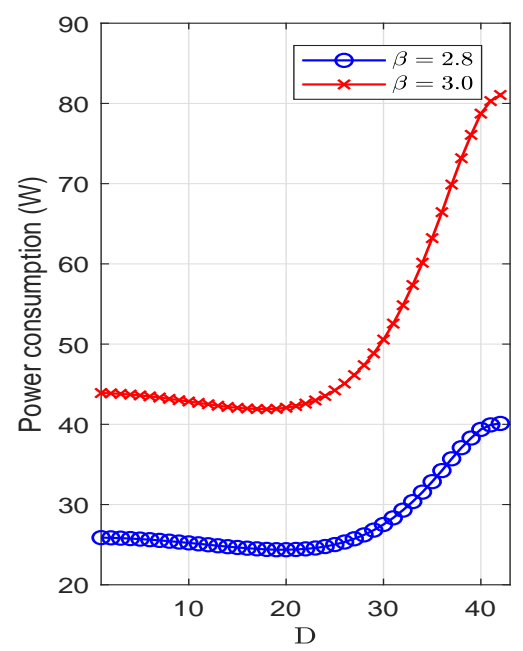

(b) $\lambda_{p}=0.002 / m^{2}, K=80$

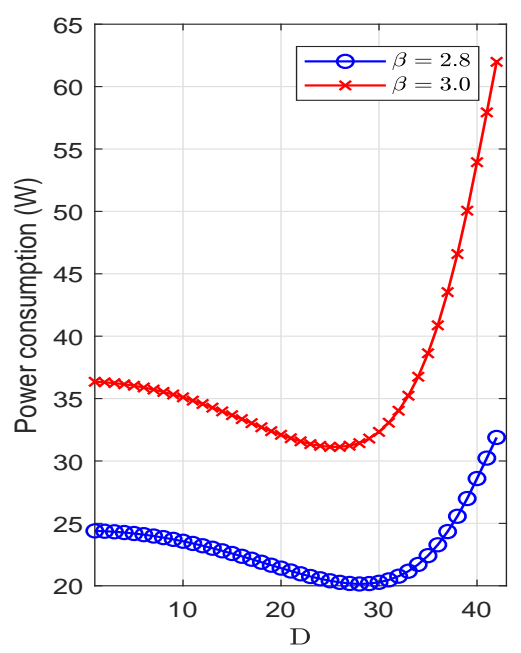

(c) $\lambda_{p}=0.003 / m^{2}, K=80$

Figure 3. Unimodality of the objective function of the problem (44).

is fully loaded to reduce the complexity of derivation. Specifically, there are at least $K_{i}$ users in the outer area of a cell and each PB serves $\bar{K}_{i}=$ $\mathbf{E}_{K i \mid K, u \in A_{\text {out }}}\left(K_{i}\right)$ UEs. The number of UEs powered by the BS is determined by $K_{0}=K \frac{D^{2}}{R^{2}}$. Then, the coverage probability conditioned on uplink and downlink transmission distances can be rewritten as

$$
C_{c}=\operatorname{Pr}\left(r_{i u}>t\right)=\mathbf{E}_{d 0 u, d i u}\left[C\left(d_{0 u}, d_{1 u}\right)\right],
$$

where $C$ is defined in (24). by

The coverage probability in the inner area is given

$$
C_{c \mid u \in A_{i n}}=\int_{0}^{D} C(r, r) f_{d_{0 u} \mid u \in A_{i n}}(r) d r
$$

which can be calculated by standard tools, such as Mathematica or Matlab.
The coverage probability in the outer area is

$$
C_{c \mid u \in A_{\text {out }}}=\int_{D}^{R} f_{d_{1 u}}(z)\left[\int_{0}^{z+R} C(r, z) f_{d_{1 u} \mid N_{1}}(r) d r\right] d z
$$

Therefore, the unconditional coverage probability can be calculated by

$C_{c}=C_{c \mid u \in A_{\text {in }}} \operatorname{Pr}\left(u \in A_{\text {in }}\right)+C_{c \mid u \in A_{\text {out }}} \operatorname{Pr}\left(u \in A_{\text {out }}\right)$.

\section{NUMERICAL RESULTS}

To evaluate the performance of the proposed method, numerical results are presented and discussed in this section. Throughout the simulations, the following settings are used unless stated otherwise. The total bandwidth is $W=1 \mathrm{MHz}$ and the density of noise power is $-144 \mathrm{dBm} / \mathrm{Hz}$. The radius of the whole area is $R=50$ meters. The average transmission power of the $\mathrm{BS}$ and a $\mathrm{PB}$ 


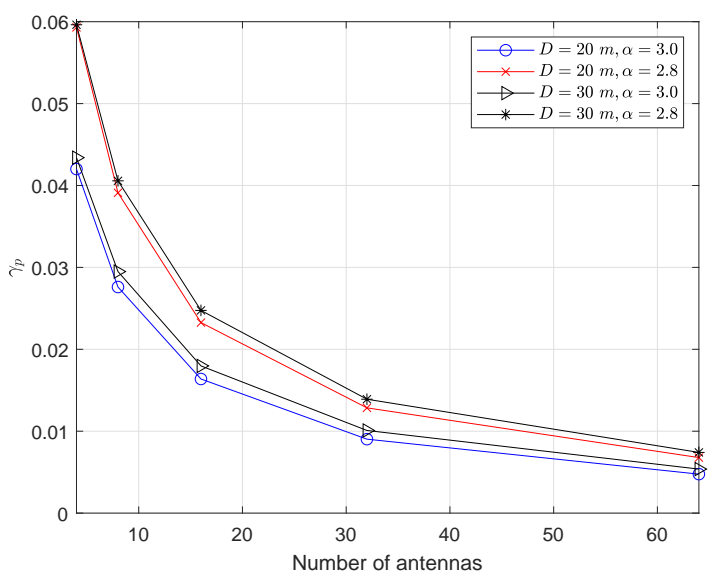

Figure 4. The ratio between the power received from non-nearest power providers and the total received power.

cannot exceed $10 \mathrm{~W}$ and $2.5 \mathrm{~W}$, respectively. Since the density of PBs in the existing literatures ranges from 0.001 to $0.1 / m^{2}[20,35]$, we set the density of PBs as $\lambda_{p}=0.003 / \mathrm{m}^{2}$. The number of transmit antennas at the BS and PBs is $N_{t}=4$. The basic power consumptions of the BS and a PB are set as $\underline{P}=2$ and $p=0.5 \mathrm{~W}$. Let the coverage probability and the required data rate be $95 \%$ and $50 \mathrm{Kbps}$. Both path loss exponents of WET and WIT are 3. The fixed path gains for WET and WIT are $\theta_{E}^{-1}=-10$ $\mathrm{dB}$ and $\theta_{I}^{-1}=-30 \mathrm{~dB}$. We set the number of UEs and $N_{1}$ as 80 and 2, respectively. The RF-DC conversion efficiency and the fraction of harvested energy used for uplink transmission are $\xi=0.7$ and $\zeta=\frac{2}{3}$, respectively.

Fig. 4 shows the ratio between the power received from non-nearest power providers and the total received power, which is denoted as $\gamma$. We can see that the amount of power received from non-nearest power providers accounts for a tiny percentage of the total received power. For example, when the number of transmit antennas increases from 4 to 64 and the path loss exponent of wireless power transfer is $\alpha=3.0, \gamma$ decreases from about $4.5 \%$ to $0.5 \%$. Therefore, receiving power from the nearest power provider is reasonable.

Figs. 5 to 6 show the performance and resource allocation of the proposed algorithm versus an increasing number of UEs (from 20 to 100 UEs). Fig. 5 compares the power consumptions for the proposed deployment method and the benchmark PB powered method (where the BS only receives information) versus the number of UEs. The pro-

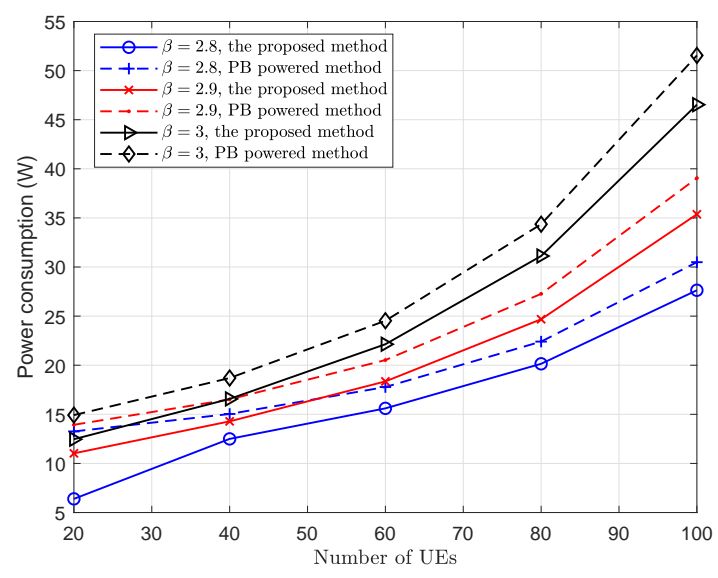

Figure 5. Total power consumption versus the number of UEs, where $\lambda_{p}=0.003 / m^{2}$.

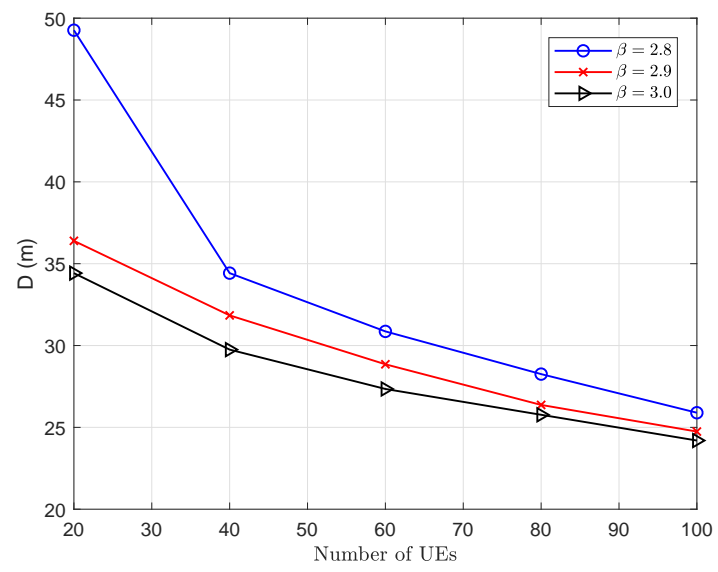

Figure 6. Radius of the inner area versus the number of UEs, where $\lambda_{p}=0.003 / m^{2}$.

posed method consumes less power than the $\mathrm{PB}$ powered method, i.e., when $\beta=3$ and $K=80$, the power consumption of the proposed method is about $31 \mathrm{~W}$, i.e., $4 \mathrm{~W}$ less than that of PB powered method. To satisfy the requirements of the coverage probability, the total power consumption increases with the number of UEs and WIT path loss exponent $\beta$. The radius of the inner area $D$ versus the number of UEs is shown in Fig. 6. The BS provides power supply for the inner UEs and it has to shrink the inner area when the number of UEs increases due to its limited transmission power, thereby decreasing $D$ to satisfy the requirement of coverage probability. This means that the outer area becomes larger and more PBs participate in power supply, which leads to a higher power consumption, as demonstrated in Fig. 5. 


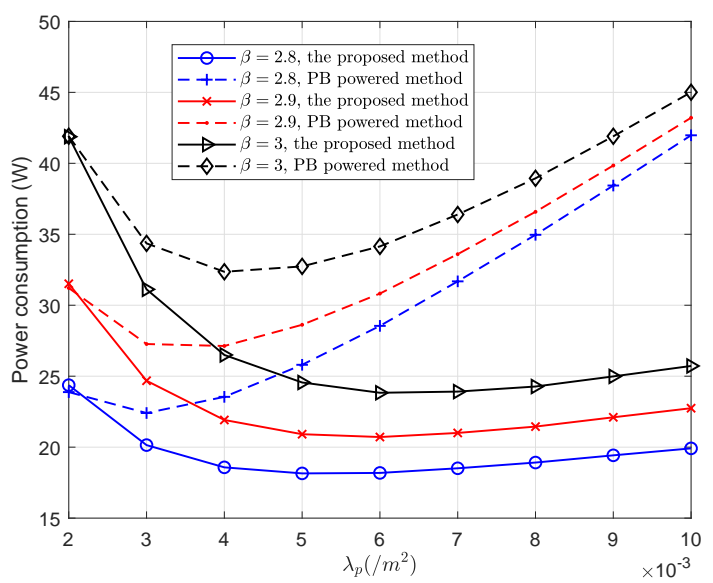

Figure 7. Total power consumption versus the density of PBs, where $K=80$.

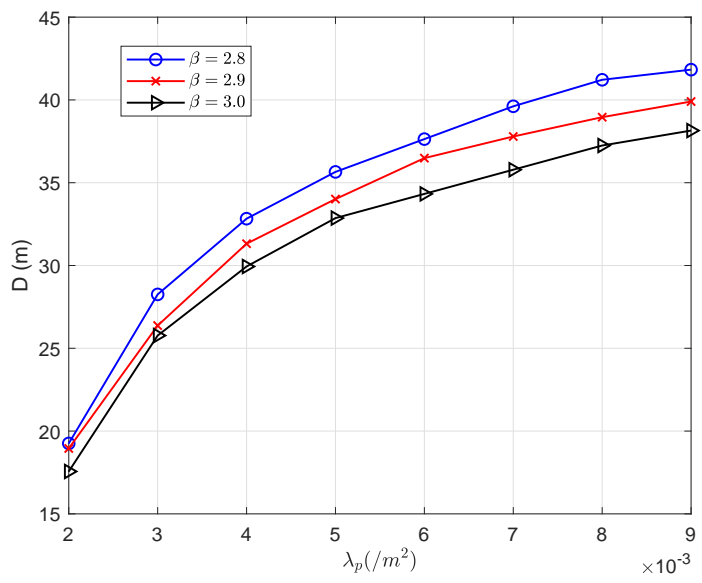

Figure 8. Radius of the inner area versus the density of PBs, where $K=80$.

Figs. 7 to 8 demonstrate the performance and resource allocation of the proposed algorithm versus the density of PBs (varying from 0.002 to $0.01 / \mathrm{m}^{2}$ ). In Fig. 7, the distance between UEs and their associated $\mathrm{PB}$ becomes shorter with an increasing density of PBs, and thus total power consumption shows a decreasing trend when $\lambda_{p}$ ranges from 0.001 to $0.006 / \mathrm{m}^{2}$ with $\beta=3$. However, the basic power consumption becomes higher when the density of PBs grows further, thereby increasing the power consumption. Besides, with a growing density of PBs, we have to extend the inner area to reduce the basic power consumption, such that the radius of the inner area increases with an increasing density of PBs, as shown in Fig. 8

Figs. 9(a) and 9(b) depict the energy efficiency versus the number of UEs and the density of PBs,

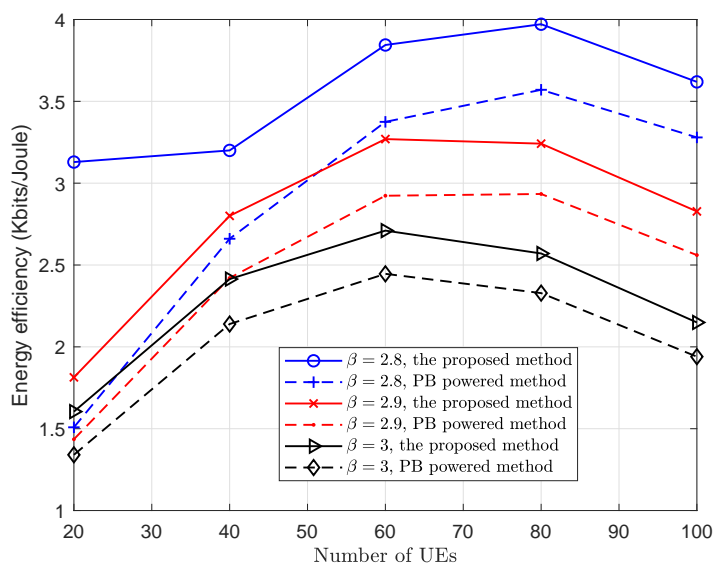

(a) Number of UEs, $\lambda_{p}=0.003 / \mathrm{m}^{2}$.

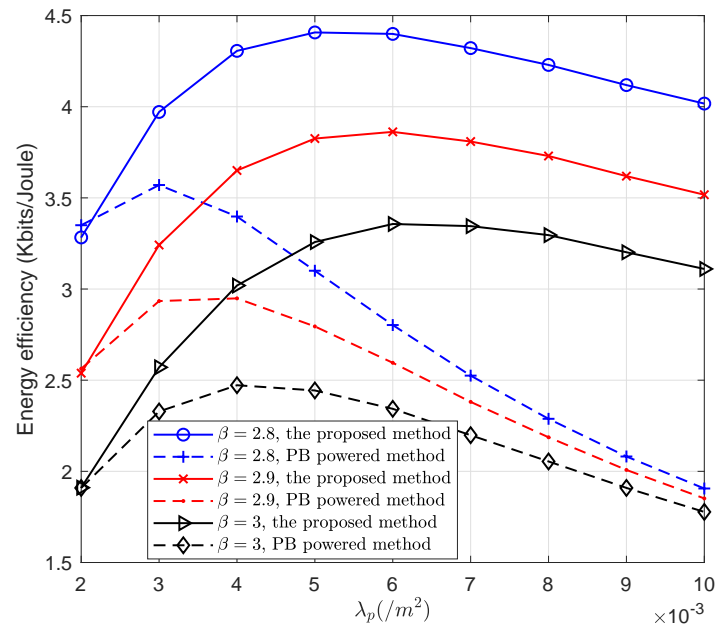

(b) Density of PBs, $K=80$.

Figure 9. Energy efficiency versus various system parameters.

respectively. In this paper, we define energy efficiency as a ratio between the sum of $K$ UEs' rate threshold $t$ and the total power consumption, which is given by

$$
E E=\frac{t K}{\mathbf{E}\left[P_{T}(D)+\underline{P}+\underline{p} \lambda_{p}\left(R^{2}-D^{2}\right)\right]} .
$$

As shown in Fig. 9(a), since the rate threshold and the number of PBs are fixed and the proportion of basic power consumption becomes lower, the energy efficiency shows a upward trend when the number of UEs changes from 20 to 60 . In contrast, the energy efficiency decreases when $K>60$, because the power consumption increases faster than the number of UEs. This can be verified in Fig. 5, where the power consumption grows dramatically when $K>60$. Besides, the energy efficiency of 


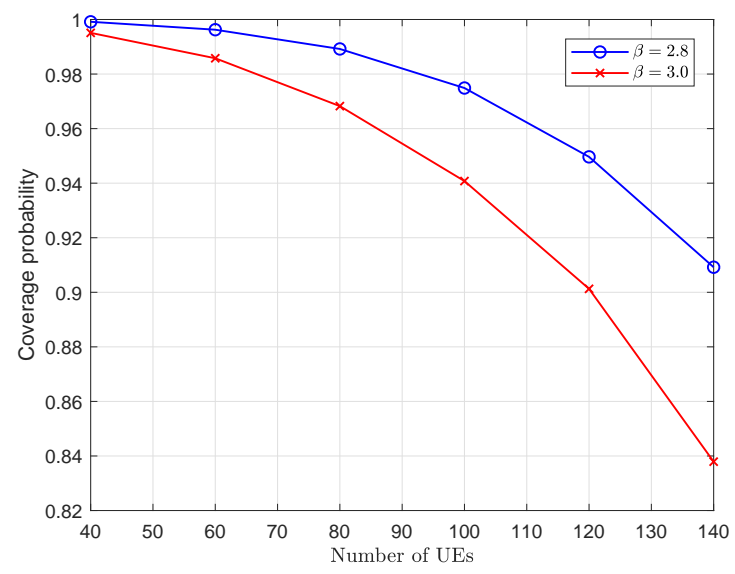

(a) Number of UEs, $\lambda_{p}=0.003 / \mathrm{m}^{2}$.

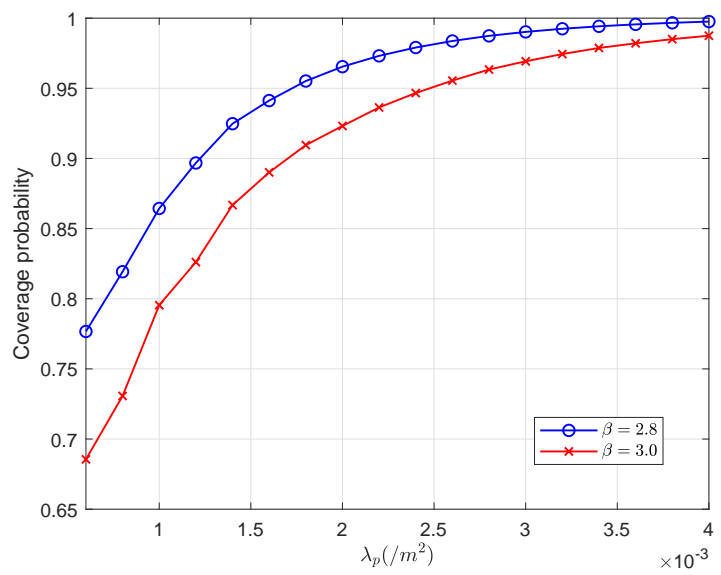

(b) Density of PBs, $K=80$.

Figure 10. Coverage probability versus various system parameters.

the proposed method outperforms that of the $\mathrm{PB}$ powered method. For instance, when $K=80$ and $\beta=3$, the energy efficiency of the proposed method is about $2.5 \mathrm{Kbits} / \mathrm{Joule}$, roughly about 0.3 Kbits/Joule higher than that of PB powered method. In Fig. 9(b), we can see that the energy efficiency shows an increasing trend first, and then decreases with a growing density of PBs, because an increasing density of PBs reduces the transmission distance of WET but increases the total basic power consumption of PBs, which is in accordance with Fig. 7.

Figs. 10(a) and 10(b) show the coverage probability versus the number of UEs and the density of PBs with different WIT path loss exponents, namely $\beta=2.8$, 3. In Fig. 10(a), the coverage probability shows a decreasing trend with a growing number of UEs. In particular, when $K$ increases from 40 to 140 with $\beta=3$, the coverage probability

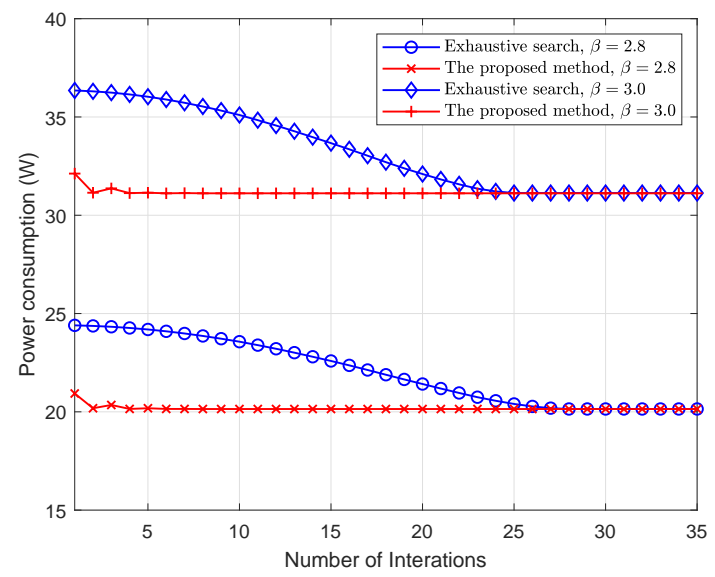

Figure 11. Average iteration numbers, where $K=80$ and $\lambda_{p}=$ $0.003 / \mathrm{m}^{2}$.

declines $16 \%$. Besides, when $K$ equals 100 , the coverage probability with $\beta=2.8$ is $0.97 \%$, about $3 \%$ higher than that of $\beta=3.0$. In Fig. 10(b), the coverage probability increases with an increasing density of PBs, because densely deployed PBs can harvest more energy for UEs' uplink information transmission. For example, when $\lambda_{p}$ changes from 0.002 to 0.003 with $\beta=3$, the coverage probability increases $4 \%$.

With a given computational accuracy (as one meter), we have compared the performances between one-dimensional search and the exhaustive search. As shown in Fig. 11, one-dimensional search converges to its optimal value within five iterations, much faster than the convergence speed of the exhaustive search.

Figs. 12(a) and 12(b) compare the power consumption under linear model, non-linear EH model, and the analytical results. The non-linear model was also used in [36]. The parameters of the non-linear model are set as $a=150, b=0.009$, and the maximal received power $M=0.02$, where the $\mathrm{EH}$ efficiency ranges from about $55 \%$ to about $78 \%$ when the input power increases from $-10 \mathrm{dBm}$ to 10 $\mathrm{dBm}$. We can observe that the amounts of received power in the linear model, non-linear EH models, and analytical results are quite close. For example, when the number of UEs equals 40 and $D=10$ $\mathrm{m}$, the amount of received power in the linear $\mathrm{EH}$ model is $21 \mathrm{~W}$, about $1 \mathrm{~W}$ and $2 \mathrm{~W}$ higher than that of the analytical results and the non-linear $\mathrm{EH}$ model, respectively. 


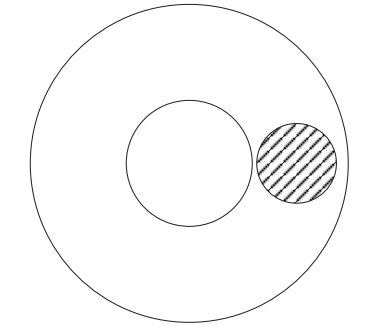

$0 \leq r \leq \min \left(d_{0 u}-D, R-d_{0 u}\right)$

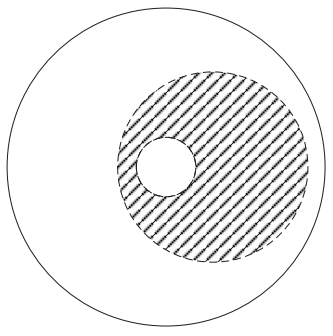

$d_{0 u}+D \leq r \leq R-d_{0 u}$

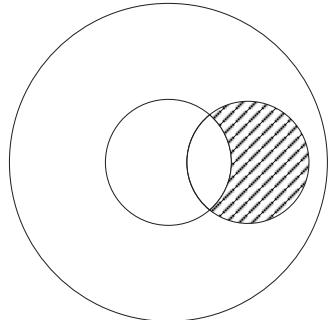

$d_{0 u}-D<r \leq R-d_{0 u}, r<d_{0 u}+D$

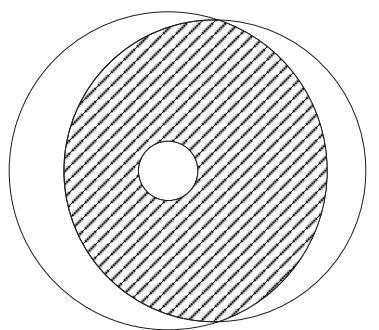

$\max \left(d_{0 u}+D, R-d_{0 u}\right) \leq r<d_{0 u}+R$

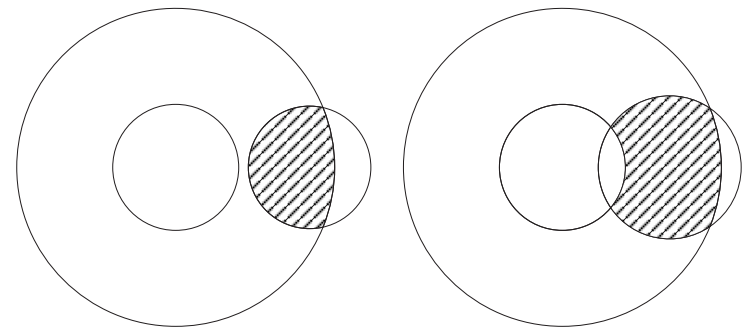

$R-d_{0 u}<r \leq d_{0 u}-D$

$\max \left(d_{0 u}-D, R-d_{0 u}\right)<r<d_{0 u}+D$

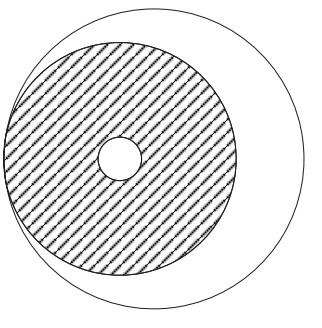

$r \geq d_{0 u}+R$

Figure 13. Interaction area $b\left(d_{0 u}, r\right)$.

\section{CONCLUSIONS}

We proposed a non-uniform deployment scheme for PBs in a WPCN, where the BS and PBs provide wireless power supply in the inner and the outer areas, respectively. Within a finite area of the outer region, the PDF of the distance from a non-centerlocated UE to its nearest $\mathrm{PB}$ is derived, conditioned on that at least $N_{1}$ PBs exist in the outer area. The total power consumption in a cell was calculated in order to satisfy UEs' coverage probability requirement. We leveraged the inner area's radius to minimize the total power consumption, and obtained a solution by adopting the SCA method. The coverage probability was derived based on the proposed method. The numerical results showed a significant power saving if compared to PB powered WPCNs, where the BS does not participate in wireless charging.

\section{APPENDIX PROOF OF THEOREM 1}

Proof: If the outer area has at least $N_{1} \mathrm{PBs}$, the complementary cumulative distribution function
(CCDF) of the nearest PB's distance is given by

$$
\begin{aligned}
& \bar{F}(r) \\
= & \operatorname{Pr}\left\{\Phi\left[b\left(d_{0 u}, r\right)\right]<1 \mid \Phi\left(A_{\text {out }}\right) \geq N_{1}\right\} \\
= & \frac{\operatorname{Pr}\left\{\Phi\left[b\left(d_{0 u}, r\right)\right]<1, \Phi\left(A_{\text {out }}\right) \geq N_{1}\right\}}{\operatorname{Pr}\left[\Phi\left(A_{\text {out }}\right) \geq N_{1}\right]} \\
\stackrel{(a)}{=} & \frac{\operatorname{Pr}\left\{\Phi\left[b\left(d_{0 u}, r\right)\right]=0\right\} \operatorname{Pr}\left\{\Phi\left[A_{\text {out }} \backslash b\left(d_{0 u}, r\right)\right] \geq N_{1}\right\}}{\operatorname{Pr}\left[\Phi\left(A_{\text {out }}\right) \geq N_{1}\right]} \\
= & \frac{e^{-\lambda_{p} \nu}\left[1-\sum_{k=0}^{N_{1}-1} \frac{e^{-\lambda_{p} \nu^{\prime}}\left(\lambda_{p} \nu^{\prime}\right)^{k}}{k !}\right]}{\sum_{k=N_{1}}^{\infty} \frac{e^{-\lambda_{p} \pi\left(R^{2}-D^{2}\right)}\left[\lambda_{p} \pi\left(R^{2}-D^{2}\right)\right]^{k}}{k !}}
\end{aligned}
$$

where equation $(a)$ holds because the number of points in the disjoint sets of a PPP are independent of each other; ")" denotes the area difference, and thus $\left[A_{\text {out }} \backslash b\left(d_{0 u}, r\right)\right]$ indicates the area defined by $\left[A_{\text {out }} \backslash b\left(d_{0 u}, r\right)\right] \bigcup b\left(d_{0 u}, r\right)=A_{\text {out }} ; \nu$ and $\nu^{\prime}$ represent the Lebesgue measures (i.e., areas) of $b\left(d_{0 u}, r\right)$ and $A_{\text {out }} \backslash b\left(d_{0 u}, r\right)$, respectively. Thus, $\nu^{\prime}$ is given by

$$
\nu^{\prime}=\pi\left(R^{2}-D^{2}\right)-\nu .
$$

The intersection areas of $b\left(d_{0 u}, r\right)$ between a circle $B\left(d_{0 u}, r\right)$ and the outer area are given in Fig. 


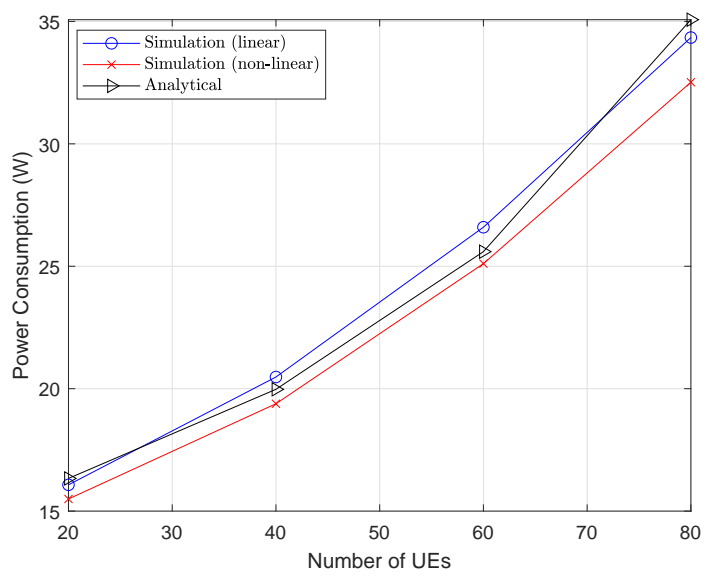

(a) $D=10 \mathrm{~m}$

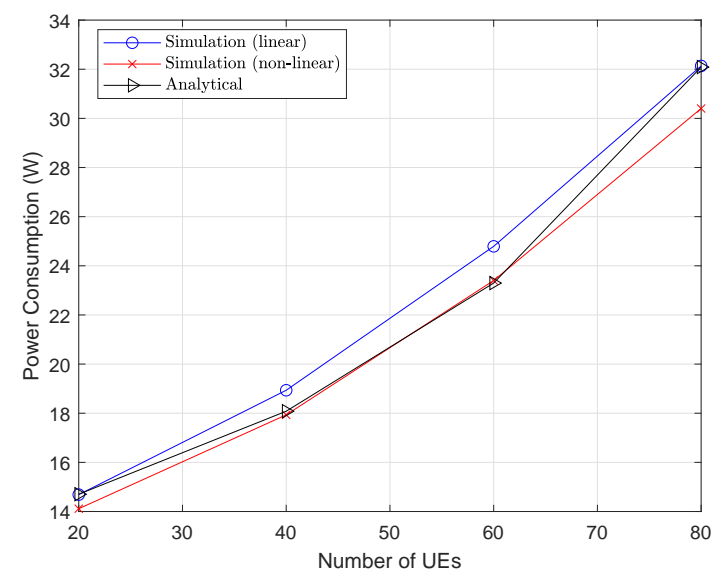

(b) $D=20 \mathrm{~m}$

Figure 12. Simulation results under linear and non-linear EH model.

13 and represented as dash areas. Therefore, $\nu$ can be obtained as (19).

\section{REFERENCES}

[1] S. Kim, R. Vyas, J. Bito, K. Niotaki, A. Collado, A. Georgiadis, and M. M. Tentzeris, "Ambient RF energy-harvesting technologies for self-sustainable standalone wireless sensor platforms," Proceedings of the IEEE, vol. 102, no. 11, pp. 1649-1666, Nov. 2014.

[2] F. Sangare, Y. Xiao, D. Niyato, and Z. Han, "Mobile charging in wireless-powered sensor networks: Optimal scheduling and experimental implementation," IEEE Transactions on Vehicular Technology, vol. 66, no. 8, pp. 7400-7410, 2017.
[3] C. H. Hyoung, J. H. Hwang, J. H. Lee, S. W. Kang, and Y. T. Kim, "Energy harvesting from electromagnetic interference induced in the human body," Electronics Letters, vol. 52, no. 22, pp. 1881-1883, Oct. 2016.

[4] L. Liu, R. Zhang, and K. C. Chua, "Multiantenna wireless powered communication with energy beamforming," IEEE Transactions on Communications, vol. 62, no. 12, pp. 43494361, Dec. 2014.

[5] G. Yang, C. K. Ho, R. Zhang, and Y. L. Guan, "Throughput optimization for massive MIMO systems powered by wireless energy transfer," IEEE Journal on Selected Areas in Communications, vol. 33, no. 8, pp. 1640-1650, Aug. 2015.

[6] K. Liang, L. Zhao, K. Yang, and X. Chu, "Online power and time allocation in MIMO uplink transmissions powered by RF wireless energy transfer," IEEE Transactions on Vehicular Technology, vol. 66, no. 8, pp. 6819-6830, 2017.

[7] E. Boshkovska, D. W. K. Ng, N. Zlatanov, A. Koelpin, and R. Schober, 'Robust resource allocation for MIMO wireless powered communication networks based on a non-linear EH model," IEEE Transactions on Communications, vol. 65, no. 5, pp. 1984-1999, 2017.

[8] L. Shi, L. Zhao, K. Liang, X. Chu, G. Wu, and H.-H. Chen, "Profit maximization in wireless powered communications with improved nonlinear energy conversion and storage efficiencies," in Proc. IEEE Int. Conf. Communications ICC 2017, Paris, France, May 21-25, 2017.

[9] P. D. Diamantoulakis, K. N. Pappi, Z. Ding, and G. K. Karagiannidis, "Wireless-powered communications with non-orthogonal multiple access," IEEE Transactions on Wireless Communications, vol. 15 , no. 12, pp. 8422-8436, Dec. 2016.

[10] F. Azmat, Y. Chen, and N. Stocks, "Predictive modeling of RF energy for wireless powered communications," IEEE Communications Letters, vol. 20, no. 1, pp. 173-176, Jan. 2016.

[11] Q. Wu, W. Chen, D. W. K. Ng, J. Li, and R. Schober, "User-centric energy efficiency maximization for wireless powered communications," IEEE Transactions on Wireless Communications, vol. 15 , no. 10, pp. 6898-6912, Jul. 2016.

[12] J. G. Andrews, F. Baccelli, and R. K. Ganti, 
"A tractable approach to coverage and rate in cellular networks," IEEE Transactions on Communications, vol. 59, no. 11, pp. 3122-3134, Oct. 2011.

[13] S. Singh, H. S. Dhillon, and J. G. Andrews, "Offloading in heterogeneous networks: Modeling, analysis, and design insights," IEEE Transactions on Wireless Communications, vol. 12, no. 5, pp. 2484-2497, Apr. 2013.

[14] H. A. Mustafa, M. Z. Shakir, M. A. Imran, and R. Tafazolli, "Spatial and social paradigms for interference and coverage analysis in underlay D2D network," IEEE Transactions on Vehicular Technology, vol. 66, no. 10, pp. 9328-9337, Apr. 2017.

[15] A. Tukmanov, S. Boussakta, Z. Ding, and A. Jamalipour, "Outage performance analysis of imperfect-CSI-based selection cooperation in random networks," IEEE Transactions on Communications, vol. 62, no. 8, pp. 2747-2757, May 2014.

[16] K. Huang and V. K. N. Lau, "Enabling wireless power transfer in cellular networks: Architecture, modeling and deployment," IEEE Transactions on Wireless Communications, vol. 13, no. 2, pp. 902-912, Feb. 2014.

[17] I. Krikidis, S. Timotheou, S. Nikolaou, G. Zheng, D. W. K. Ng, and R. Schober, "Simultaneous wireless information and power transfer in modern communication systems," IEEE Communications Magazine, vol. 52, no. 11, pp. 104-110, Nov. 2014.

[18] H. Tabassum and E. Hossain, "On the deployment of energy sources in wireless-powered cellular networks," IEEE Transactions on Communications, vol. 63, no. 9, pp. 3391-3404, Sep. 2015.

[19] C. Zhang and G. Zhao, "Wireless powered sensor networks with random deployments," IEEE Wireless Communications Letters, vol. 6, no. 2, pp. 218-221, Apr. 2017.

[20] Z. Wang, L. Duan, and R. Zhang, "Adaptively directional wireless power transfer for largescale sensor networks," IEEE Journal on Selected Areas in Communications, vol. 34, no. 5, pp. 1785-1800, Apr. 2016.

[21] O. Galinina, H. Tabassum, K. Mikhaylov, and S. Andreev, "On feasibility of 5G- grade dedicated RF charging technology for wirelesspowered wearables," IEEE Wireless Communi- cations, vol. 23, no. 2, pp. 28-37, 2016.

[22] M. Haenggi, "On distances in uniformly random networks," IEEE Transactions on Information Theory, vol. 51, no. 10, pp. 3584-3586, 2005.

[23] S. Srinivasa and M. Haenggi, "Distance distributions in finite uniformly random networks: Theory and applications," IEEE Transactions on Vehicular Technology, vol. 59, no. 2, pp. 940949, 2010.

[24] M. Afshang and H. S. Dhillon, "Fundamentals of modeling finite wireless networks using binomial point process," IEEE Transactions on Wireless Communications, vol. 16, no. 5, pp. 3355-3370, 2017.

[25] A. Tukmanov, S. Boussakta, Z. Ding, and A. Jamalipour, "On the impact of relay-side channel state information on opportunistic relaying," 2013 IEEE International Conference on Communications (ICC), Budapest, pp. 54785482, 2013.

[26] D. Stoyan, W. S. Kendall, and J. Mecke, "Stochastic geometry and its applications, 2nd ed." Wiley, 2008.

[27] H. Ju and R. Zhang, "Throughput maximization in wireless powered communication networks," IEEE Transactions on Wireless Communications, vol. 13, no. 1, pp. 418-428, Dec. 2014.

[28] S. Cai, Y. Che, L. Duan, J. Wang, S. Zhou, and R. Zhang, "Green 5G heterogeneous networks through dynamic small-cell operation," IEEE Journal on Selected Areas in Communications, vol. 34, no. 5, pp. 1103-1115, Jan. 2016.

[29] X. Chen, C. Yuen, and Z. Zhang, "Wireless energy and information transfer tradeoff for limited-feedback multiantenna systems with energy beamforming," IEEE Transactions on Vehicular Technology, vol. 63, no. 1, pp. 407412, 2014

[30] R. Zhang and C. K. Ho, "Mimo broadcasting for simultaneous wireless information and power transfer," IEEE Transactions on Wireless Communications, vol. 12, no. 5, pp. 1989-2001, 2013.

[31] R. E. Barnett, J. Liu, and S. Lazar, "A RF to DC voltage conversion model for multi-stage rectifiers in UHF RFID transponders," SolidState Circuits, IEEE Journal of, vol. 44, no. 2, pp. 354-370, Jan. 2009. 
[32] H. Ju and R. Zhang, "Optimal resource allocation in full-duplex wireless-powered communication network," Communications IEEE Transactions on, vol. 62, no. 10, pp. 3528-3540, 2014.

[33] R. W. Heath, T. Wu, Y. H. Kwon, and A. C. K. Soong, "Multiuser mimo in distributed antenna systems with out-of-cell interference," IEEE Transactions on Signal Processing, vol. 59, no. 10, pp. 4885-4899, 2011.

[34] J. S. Ferenc and Z. Nda, "On the size distribution of poisson voronoi cells," Physica A Statistical Mechanics \& Its Applications, vol. 385, no. 2, pp. 518-526, Nov. 2007.

[35] Y. L. Che, L. Duan, and R. Zhang, "Spatial throughput maximization of wireless powered communication networks," IEEE Journal on Selected Areas in Communications, vol. 33, no. 8, pp. 1534-1548, 2014.

[36] E. Boshkovska, D. W. K. Ng, N. Zlatanov, and R. Schober, "Practical non-linear energy harvesting model and resource allocation for swipt systems," IEEE Communications Letters, vol. 19, no. 12, pp. 2082-2085, 2015. 